\title{
Syndrome of Extreme Insulin Resistance (Rabson-Mendenhall Phenotype) with Atrial Septal Defect: Clinical Presentation and Treatment Outcomes
}

\author{
Deep Dutta, Indira Maisnam, Sujoy Ghosh, Satinath Mukhopadhyay, Subhankar Chowdhury \\ IPGMER \& SSKM Hospital, Department of Endocrinology \& Metabolism, Kolkata, India
}

\begin{abstract}
Syndrome of extreme insulin resistance (SEIR) is a rare spectrum disorder with a primary defect in insulin receptor signalling, noted primarily in children, and is often difficult to diagnose due to the clinical heterogeneity. SEIR was diagnosed in an adolescent girl with facial dysmorphism, exuberant scalp and body hair, severe acanthosis, lipoatrophy, dental abnormalities, and short stature (Rabson-Mendenhall phenotype). She had elevated fasting (422.95 pmol/L) and post-glucose insulin levels (>2083 pmol/L). Total body fat was decreased (11\%; dual-energy X-ray absorptiometry). Basal growth hormone (GH) was increased (7.9 $\mu \mathrm{g} / \mathrm{L})$ with normal insuline-like growth factor $1(37.6 \mathrm{nmol} / \mathrm{L})$ suggestive of $\mathrm{GH}$ resistance. She had fatty liver and polycystic ovaries. Echocardiography revealed ostium secundum type atrial septal defect (ASD). Blood glucose normalized with pioglitazone (30 mg/day). Delayed development, severe insulin resistance, mild hyperglycemia, absence of ketosis, and remarkable response of hyperinsulinemia and hyperglycemia to pioglitazone which persisted even after 1 year of diagnosis are some of the notable features of this patient. This is perhaps the first report of occurrence of congenital heart disease (ASD) in a patient of SEIR (Rabson-Mendenhall phenotype). This report highlights the clinical features of SEIR and the role of insulin sensitizers like pioglitazone in the management of such patients.
\end{abstract}

Key words: Rabson-Mendenhall syndrome, insulin resistance, lipoatrophy, atrial septal defect, short stature

Conflict of interest: None declared

Received: 04.10 .2012

Accepted:02.12.2012

\section{Introduction}

Syndromes of extreme insulin resistance (SEIR) represent an extreme end of a spectrum disorder of insulin resistance due a defect either in the insulin receptor or downstream in the insulin signalling pathway [Leprechaunism, Rabson-Mendenhall syndrome (RMS), Type-A syndrome, lipoatrophic diabetes], or auto antibodies to insulin receptor/insulin. Hyperinsulinemia and differential resistance to insulin action in different tissues and organs explain most of the manifestations of these disorders.

Leprechaunism is the most severe of these conditions leading to death usually within the first year of life. RMS is a less severe form which can be differentiated from leprechaunism by presence of teeth and nail abnormalities, coarse facial features, acanthosis, pineal hyperplasia and survival till adolescence (1). We present an adolescent with SEIR who had RMS phenotype with atrial septal defect (ASD).

\section{Case Report}

A thirteen-year-old girl presented with poor height and weight gain, lack of development of secondary sexual features, primary amenorrhea, and skin hyperpigmentation. She was born to non-consanguineous parents at term with a birth weight of $1.5 \mathrm{~kg}$ and had delayed milestones, including delayed dentition. She had clitoromegaly since birth for which she underwent a reductive surgery at age 2 years. Examination revealed a birdlike facies, pinched up pointed nose, hollow cheeks (Figure 1),

\section{Address for Correspondence}

Deep Dutta MD, IPGMER \& SSKM Hospital, Department of Endocrinology \& Metabolism, Kolkata, India Phone: +919477406630 E-mail: deepdutta2000@yahoo.com

@ Journal of Clinical Research in Pediatric Endocrinology, Published by Galenos Publishing. 
prominent mandible, abnormal dentition (Figures 2, 3), exuberant scalp and body hair, acanthosis over the nape of her neck, on the axilla, elbows and knuckles, a shrivelled skin mainly in her upper limbs and trunk (Figure 4). Her height was $130 \mathrm{~cm}\left(<3^{\text {rd }}\right.$ percentile; standard deviation score (SDS): -3.04), weight $20 \mathrm{~kg}$ $\left(<3^{\text {rd }}\right.$ percentile; weight SDS: -2.32$)$, and her body mass index was $11.83 \mathrm{~kg} / \mathrm{m}^{2}$ (<3rd percentile). Mid-parental height was 147 $\mathrm{cm}\left(<3^{\text {rd }}\right.$ percentile; SDS: -2.28$)$. Sexual maturity rating was prepubertal. External genital examination revealed an enlarged but mutilated clitoris (clitoral index: $60 \mathrm{~mm}^{2}$; normal <35 mm2).

Her karyotype was 46XX. She had fasting and post-glucose (following $35 \mathrm{~g}(1.75 \mathrm{~g} / \mathrm{kg})$ of glucose for a glucose tolerance test) hyperglycemia, hyperinsulinemia, and an increased C-peptide level (Table 1). Serum beta-hydroxybutyrate level was normal (Table 2) and urine ketones were negative. She had a raised serum testosterone level $(0.067 \mathrm{nmol} / \mathrm{L})$. Bone age was 13 years (Greulich-Pyle method). Abdominal ultrasonography showed normal kidneys and Mullerian structures, a liver with heterogeneous echotexture, and polycystic ovaries with echogenic stroma (Figure 5). Dual-energy X-ray absorptiometry (DEXA) showed reduced total body fat (11.3\%; normal 15$25 \%$ ). Echocardiography showed small ostium secundum ASD
(Figure 6). Brain magnetic resonance imaging (MRI) showed a normal pineal gland (Figure 7).

Treatment was initiated with pioglitazone 15 mg/day, increased to $30 \mathrm{mg} /$ day after 6 weeks. Last evaluated after 1 year of initial diagnosis, her blood glucose is well controlled [fasting blood sugar glucose: $5.11 \mathrm{mmol} / \mathrm{L}$; postprandial blood sugar glucose: $7.33 \mathrm{mmol} / \mathrm{L}$; hemoglobin A1c (HbA1c): 0.069], she had no history of hypoglycaemia, her acanthosis had improved, and she had advanced into puberty (breast stage: B2, pubic hair stage: P3).

\section{Discussion}

Both alleles of insulin receptor are abnormal in RMS and these patients fail to respond to endogenous as well as exogenous insulin (2). The natural history of a patient of RMS is marked by fasting hypoglycaemia and postprandial hyperglycemia in the first year of life, followed by constant hyperglycemia (by 3-4 years age) and ketoacidosis usually by 6-7 years of age. Most patients die of severe intractable ketoacidosis by adolescence. It has been shown that there is a rapid fall in serum insulin levels in patients with RMS

Table 1. Changes in blood sugar, insulin, and C-peptide during the oral glucose tolerance test (OGTT)

\begin{tabular}{lccc}
\hline Parameter & Fasting & OGTT (with $\mathbf{3 5}$ g glucose) \\
1 hour post-glucose & 2 hours post-glucose \\
\hline Blood glucose (mmol/L) & 7.83 & 15.65 & 13.04 \\
Insulin (pmol/L) & 422.95 & $>2083$ & $>2083$ \\
C-peptide (nmol/L) & 1.98 & 6.06 & - \\
\hline$*: 1.75 \mathrm{~g} / \mathrm{kg}$ & & & \\
\hline
\end{tabular}

Table 2. Baseline laboratory investigations

\begin{tabular}{|c|c|c|c|}
\hline Parameter & & Parameter & \\
\hline $\mathrm{LH}(\mathrm{IU} / \mathrm{L})^{\#}$ & 1.42 & Total cholesterol (mmol/L) & 3.03 \\
\hline FSH (IU/L)\# & 3.56 & Triglyceride (mmol/L) & 2.09 \\
\hline DHEA-S (nmol/L) & 245.11 & LDL-C (mmol/L) & 1.30 \\
\hline Prolactin (pmol/L) & 162.6 & HDL-C (mmol/L) & 0.96 \\
\hline fT4 (pmol/L) & 15.96 & Calcium (mg/dL) & 2.73 \\
\hline TSH (mIU/L) & 3.4 & Phosphorus (mg/dL) & 1.59 \\
\hline Cortisol ( $\mu \mathrm{g} / \mathrm{dL})$ (8am) & 386.26 & AST (U/L) & 35 \\
\hline Basal GH $(\mu \mathrm{g} / \mathrm{L})^{\#}$ & 7.9 & $A L P(U / L)$ & 29 \\
\hline IGF-1 (nmol/L) (23.97-111.35) & 37.60 & $\mathrm{Na}^{+}(\mathrm{mmol} / \mathrm{L})$ & 142 \\
\hline 17-OH progesterone (nmol/L) & 4.45 & $\mathrm{BHB}(\mathrm{mmol} / \mathrm{L})$ & 0.1 \\
\hline 25(OH)D (nmol/L) & 119.31 & $\mathrm{HbA1c}$ & 0.072 \\
\hline
\end{tabular}

\#: 3 pooled samples 30 minutes apart

LH: luteinizing hormone, FSH: follicle-stimulating hormone, DHEA-S: dehydroepiandrosterone-sulfate, fT4: free thyroxine, TSH: thyroid-stimulating hormone, GH: growth hormone, IGF-1: insulin-like growth factor 1, 25(OH)D: 25-hydroxy vitamin-D, LDL-C: low-density lipoprotein cholesterol, HDL-C: high-density lipoprotein cholesterol,

AST: aspartate aminotransferase, ALP: alkaline phosphatase, $\mathrm{Na}^{+}$: sodium, BHB: beta-hydroxybutyrate 
beyond 2 years of age, with maintained glucagon which may explain the ketosis. Infusion of very high doses of insulin 19.5 $\mathrm{U} / \mathrm{kg} / \mathrm{hour}$ ) or high doses of insulin-like growth factor 1 (IGF1) $(1.6 \mathrm{mg} / \mathrm{kg} /$ day $)$ have been shown to block ketone body production and control fasting hyperglycemia $(2,3)$. High doses of metformin and glitazones (troglitazone, rosiglitazone) have been tried in patients with RMS with varied response (4). Recombinant methionyl human leptin (r-metHuLeptin) has been reported to be useful in controlling hyperglycemia, insulin levels, and $\mathrm{HbA1c}$ in patients with RMS and other lipodystrophy syndromes $(4,5)$.

RMS was suspected in this patient because of the classical facial features, presence of exuberant scalp hair and hirsutism, typical dental abnormalities, growth retardation,

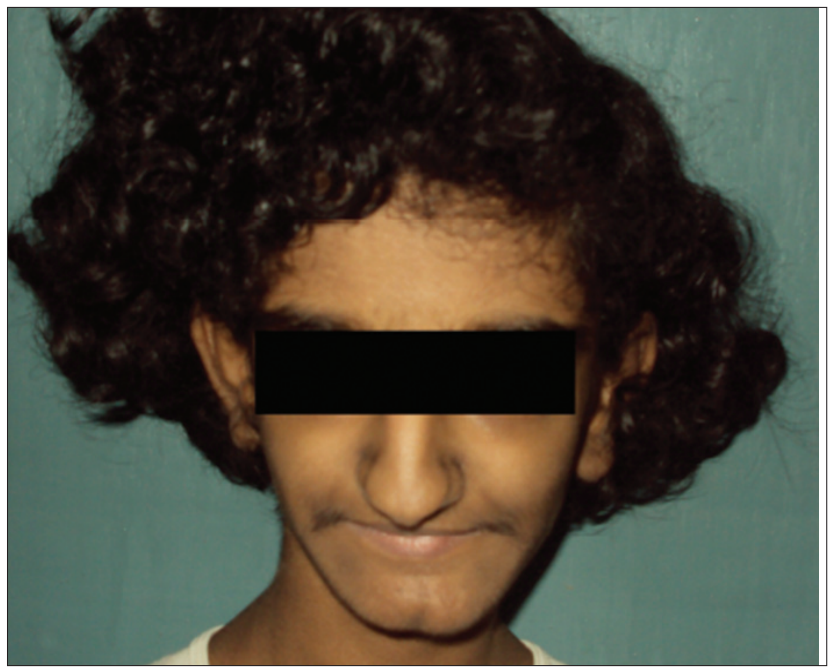

Figure 1. Facial profile of the patient showing pinched up nose, hollow cheeks, exuberant scalp hair, and increased terminal hair on face

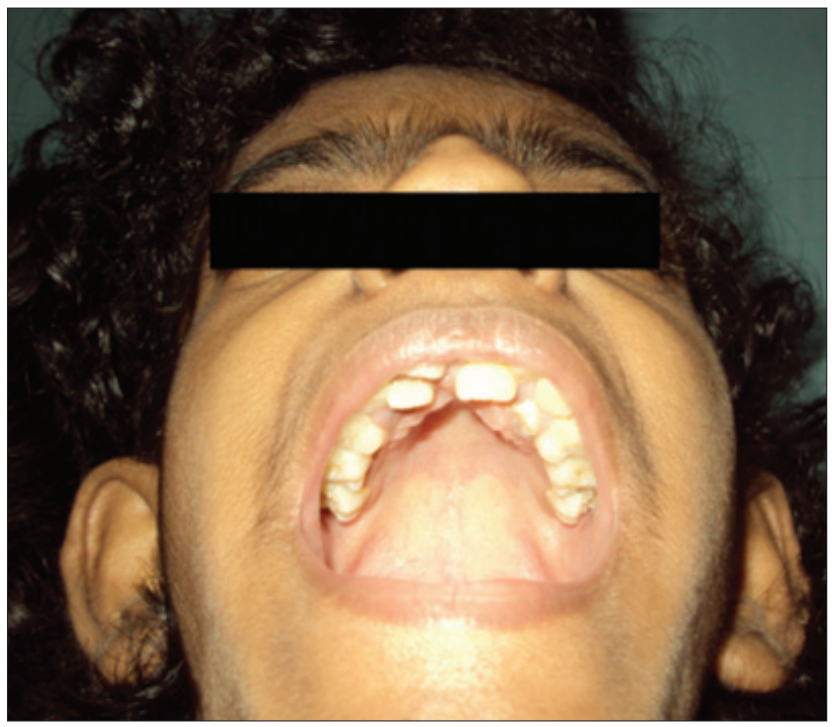

Figure 2. Upper jaw of the patient showing two rows of teeth acanthosis nigricans, lack of subcutaneous fat (lipoatrophy), hyperandrogenism, enlargement of external genitalia (clitoromegaly), extremely elevated levels of circulating insulin

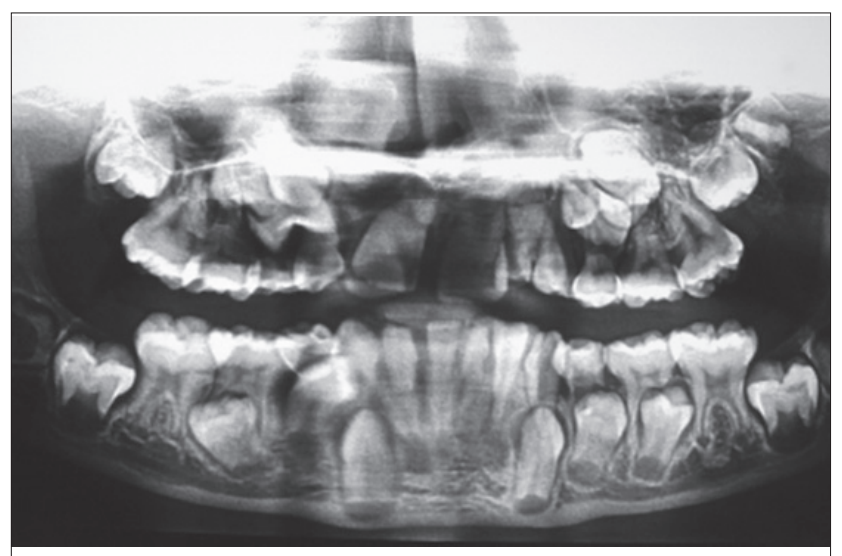

Figure 3. Orthopantogram showing two rows of teeth in upper and lower jaw

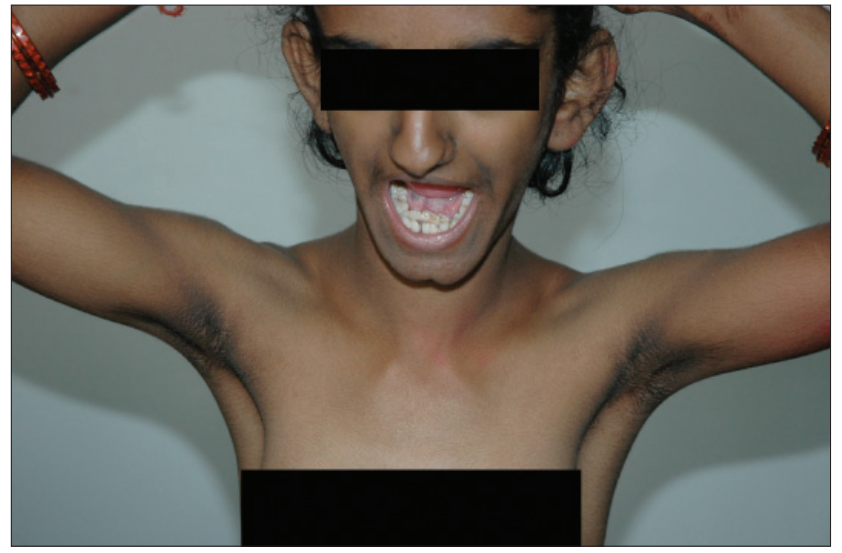

Figure 4. Profile of the patient showing two rows of teeth in the lower jaw, increased terminal hair on the face, along with acanthosis around the neck and axilla

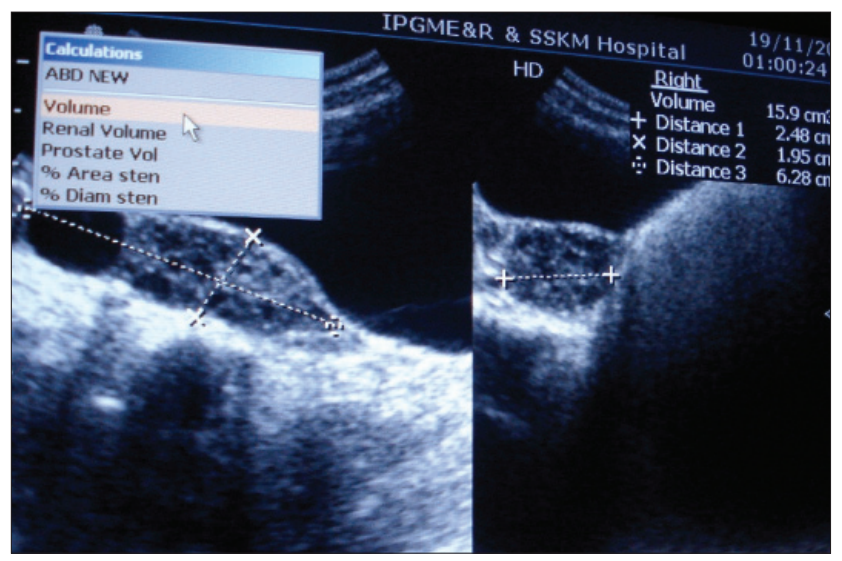

Figure 5. Ultrasonography of the abdomen showing bilateral polycystic ovaries with presence of a dominant cyst in the right ovary 


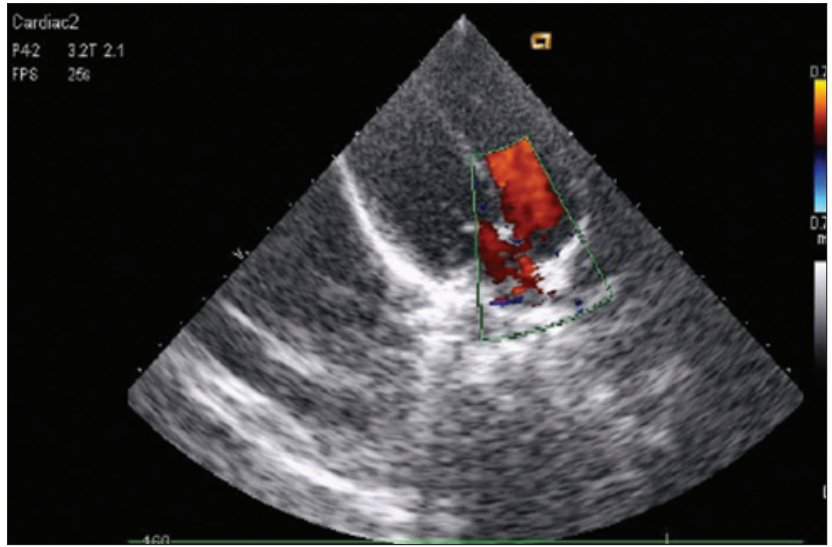

Figure 6. Echocardiography showing ostium secundum variant of atrial septal defect

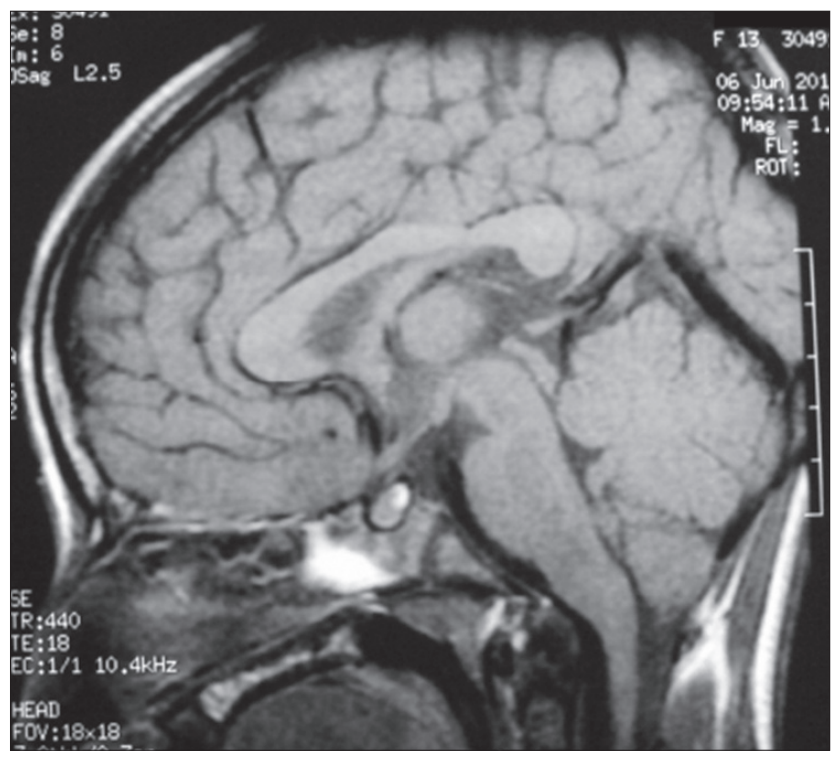

Figure 7. Magnetic resonance imaging of brain (T1W) showing normal pituitary and suprasellar region with normal pineal gland

and also presence of other supportive biochemical parameters. Our patient was born small for gestational age, which is commonly seen in RMS. She had polycystic ovaries and fatty liver, and her lipid profile was typical of metabolic syndrome. Decreased total body fat on DEXA confirms the lipoatrophic phenotype. RMS is often associated with precocious puberty, in contrast to the findings of our patient. Presentation with delayed development and with mild hyperglycemia in our patient can be explained by the heterogeneity of mutations in the insulin receptor.

This is the first report of occurrence of a congenital heart disease in a patient of SEIR with RMS phenotype. Heart diseases are uncommon in patients of lipoatrophic syndrome except for Berardinelli-Seip congenital lipodystrophy where hypertrophic cardiomyopathy is rarely reported in the third decade of life.
Counter-regulatory hormones have an important role in the pathogenesis of hyperglycemia in patients with RMS (6). Studies have shown normal to elevated growth hormone (GH) levels in patients with RMS $(6,7)$ with transient improvement in metabolic parameters following hypophysectomy in a patient with RMS (8). Our patient had an elevated basal GH level but normal IGF-1 level, as also reported previously (9). However, recombinant GH and IGF-1 are not useful in increasing height in patients with RMS due to resistance to their action $(4,9)$. Our patient has decreased whole body fat on DEXA, a finding which is expected in the broader group of lipoatrophic syndromes and also seen in RMS.

One of the limitations of this case report is the lack of study for mutation in the insulin receptor gene. Though uncommon, SEIR should always be considered in a child with suspicious phenotype, and the patient's insulin resistance should be evaluated. Insulin sensitizers have an important role in the management of these patients, in contrast to insulin, which may be ineffective. To summarize, we presented an adolescent with SEIR, RMS phenotype, lipoatrophy, and ASD who responded to pioglitazone therapy.

\section{References}

1. Goldfine $A B$, Moses AC. Syndromes of extreme insulin resistance. In: Kahn CR, Weir GC, et al. Joslin's Diabetes Mellitus, 14th ed. 2005;28: 493-498.

2. Longo N, Wang $Y$, Pasquali M. Progressive decline in insulin levels in Rabson-Mendenhall syndrome. J Clin Endocrinol Metab 1999;84:2623-2629.

3. Nakae J, Kato M, Murashita M, Shinohara N, Tajima T, Fujieda K. Long-term effect of recombinant human insulin-like growth factor I on metabolic and growth control in a patient with leprechaunism. J Clin Endocrinol Metab 1998;83:542-549.

4. Musso C, Cochran E, Moran SA, Skarulis MC, Oral EA, Taylor $S$, Gorden P. Clinical course of genetic diseases of the insulin receptor (type $\mathrm{A}$ and Rabson-Mendenhall syndromes): a 30-year prospective. Medicine (Baltimore) 2004;83:209-222.

5. Cochran E, Young JR, Sebring N, DePaoli A, Oral EA, Gorden P. Efficacy of recombinant methionyl human leptin therapy for the extreme insulin resistance of the Rabson-Mendenhall syndrome. J Clin Endocrinol Metab 2004;89:1548-1554.

6. D'Ercole AJ, Underwood LE, Groelke J, Plet A. Leprechaunism: studies of the relationship among hyperinsulinism, insulin resistance, and growth retardation. J Clin Endocrinol Metab 1979;48:495-502.

7. Kobayashi M, Takata Y, Sasaoka T, Shigeta Y, Goji K. Fluctuation of insulin resistance in a leprechaun with a primary defect in insulin binding. J Clin Endocrinol Metab 1988;66:1084-1088.

8. West RJ, Leonard JV. Familial insulin resistance with pineal hyperplasia: metabolic studies and effect of hypophysectomy. Arch Dis Child 1980;55:619-621.

9. Longo N, Singh R, Griffin LD, Langley SD, Parks JS, Elsas LJ. Impaired growth in Rabson-Mendenhall syndrome: lack of effect of growth hormone and insulin-like growth factor-I. J Clin Endocrinol Metab 1994;79:799-805. 